\title{
Effects of Stereoisomeric Isoleucines on Sporidesmolide Biosynthesis by Pithomyces chartarum
}

\author{
By D. W. RUSSELL* \\ Twyford Laboratories, Twyford Abbey Road, London, N.W. 10
}

(Accepted for publication 31 December 1966)

\begin{abstract}
SUMMARY
As well as the known sporidesmolides I, II and III, Pithomyces chartarum produces sporidesmolides or similar compounds which contain residues of erythro-isoleucine and $\alpha$-hydroxyisocaproic acid. When erythro-L-isoleucine was added to the growth medium, synthesis of sporidesmolides I and III was inhibited and synthesis of isoleucine-containing sporidesmolides, mainly sporidesmolide II, was promoted. Threo-L-isoleucine in the medium was poorly utilized, but its presence resulted in production of a very complex mixture of sporidesmolides containing a higher proportion of isoleucine residues than the control. Epimerization of erythro-D-isoleucine occurred in the medium. Neither epimer was well utilized, and this isomer had little effect on sporidesmolide production or composition; it promoted some increase in the synthesis of isoleucine-containing sporidesmolides. Threo-Disoleucine had effects qualitatively similar to but less pronounced than those of erythro-L-isoleucine, probably because of extracellular epimerization. The use of epimeric pairs of amino acids as tools for investigating biochemical processes involving inversion of configuration at an amino acid $\alpha$-carbon atom is suggested.
\end{abstract}

\section{INTRODUCTION}

This paper describes some biochemical effects of $\alpha$-amino- $\beta$-methyl-valeric acid (1), the trivial name for which is isoleucine. The $\alpha$ - and $\beta$-carbon atoms are both asymmetrically substituted, so that the amino acid exists in four stereoisomeric forms:<smiles>CCC(C)C([NH3+])C(=O)[O-]</smiles>

These are distinguished by the prefixes L- or D-, referring to the configuration about the $\alpha$-carbon atom, and also by the prefixes threo- and erythro-, referring to the configurational relationship between the $\alpha$ - and $\beta$-carbon atoms (Vickery, 1963). Thus, the isomer that is a common protein constituent is erythro-L-isoleucine which, by inversion at the $\alpha$-carbon atom only, is converted to its epimer, threo-D-isoleucine. In this paper the term 'isoleucine' is used without qualification when the stereochemical configuration is unspecified.

* Present address: Department of Biochemistry, Dalhousie University, Halifax, Nova Scotia. 
The fungus Pithomyces chartarum (Berk. \& Curt.) M. B. Ellis (Ellis, 1960) produces a mixture of insoluble cyclodepsipeptides termed sporidesmolides (Done, Mortimer, Taylor \& Russell, 1961) which are located as spicules on the surface of the spores (Bertaud, Morice, Russell \& Taylor, 1963). Four pure sporidesmolides have been isolated.

$$
\begin{gathered}
\mathrm{L}-\mathrm{Me}_{2} \mathrm{CH} .\left(\mathrm{CH}_{2}\right)_{n} \cdot \mathrm{CH} \cdot \mathrm{CO} \longrightarrow \mathrm{L}-\mathrm{Val} \longrightarrow \mathrm{A} \longrightarrow \mathrm{O} \\
1, n=0, \mathrm{~A}=\mathrm{L}-\mathrm{Meleu}, \mathrm{B}=\mathrm{D}-\mathrm{Val}: \\
2, n=0, \mathrm{~A}=\mathrm{L}-\mathrm{Meleu}, \mathrm{B}=\text { threo-D-Ile } \\
3, n=0, \mathrm{~A}=\mathrm{L}-\mathrm{Ceu}, \quad \mathrm{B}=\mathrm{D}-\mathrm{Cal}, \\
4, n=1, \mathrm{~A}=\mathrm{L}-\mathrm{CHMeleu}, \mathrm{B}=\mathrm{D}-\text { Val. }
\end{gathered}
$$

When $P$. chartarum was grown in liquid surface culture on potato + carrot extract it formed mainly sporidesmolide I. This was shown by chemical degradation to be 1 , a structure which has since been confirmed by synthesis (Shemyakin et al. 1965). A second component was also present which, although it was not isolated, was named sporidesmolide II and was tentatively assigned a structure in which the $\mathrm{D}$-valine residue of 1 was replaced by a residue of a D-isoleucine isomer (Russell, $1960 a$, 1962). When $P$. chartarum was grown on potato + carrot extract to which optically inactive isoleucine had been added, its production of sporidesmolide I was inhibited, and the only compound that could be isolated was 2 for which in this paper the name sporidesmolide II is retained (Bertaud, Probine, Shannon \& Taylor, 1965). Sporidesmolide II has been synthesized (Shemyakin et al. 1965), thus providing further confirmation that the isoleucine residue has the threo-D-configuration. A third compound, sporidesmolide III, is formed in small amount by $P$. chartarum growing on potato + carrot extract, and was shown to have structure 3 (Russell, MacDonald \& Shannon, 1964). Sporidesmolide IV has not so far been obtained from $P$. chartarum, but is produced by the closely related P. maydicus (Sacc.) M. B. Ellis, (Ellis, 1960: Bishop et al. 1965). Its structure 4 was deduced by degradation (Bishop \& Russell, $1964,1967)$ and, together with that of sporidesmolide III, was confirmed by synthesis (Ovchinnikov, Kiryushkin \& Shemyakin, 1966).

The aim of the experiments to be described was to determine the effects of each separate stereoisomer of isoleucine on the biosynthesis of sporidesmolides I, II and III. During the course of this work it became clear that these were not the only sporidesmolides produced by this organism, a conclusion also reached by Bertaud et al. (1965). Further work on this problem is clearly necessary. The present author is no longer working with sporidesmolides, but the results obtained so far appear to be interesting and are presented here.

\section{METHODS}

Organism. An isolate of Pithomyces chartarum derived from isolate 'C' (Done et al. 1961) was used. During the 4 years over which these experiments were conducted, the organism was maintained at $25^{\circ}$ on potato glucose agar slopes by repeated subculture. From time to time it lost its ability to sporulate freely, and single-spore subculture from a vigorously sporing sector was used to obtain a suitably high-sporing isolate. It is therefore unlikely that the organism is now identical with isolate ' $\mathrm{C}$ ', although the cyclodepsipeptides that it produces are unchanged. 
Cultivation of organism. In one experiment the salts + glucose + asparagine medium of Ross (1960; Butler, Russell \& Clarke, 1962) supplemented with yeast extract $(0.1 \%$, w/v; ' Difco' brand), was used. In all others the medium was the potato + carrot extract of Done et al. (1961), containing dry matter $2 \%(\mathrm{w} / \mathrm{v})$, reducing sugars $1 \%(\mathrm{w} / \mathrm{v}$; adjusted with glucose) and nitrogen $0.05 \%$ (w/v; adjusted with ammonium sulphate). Amino acids were added before autoclaving. The methods used for sterilization of media and preparation of inocula (Done et al. 1961), and the general conditions of cultivation (Bishop et al. 1965) were as described.

Amino acids. Erythro- and threo-L-isoleucine were from Mann Research Laboratories, New York, N.Y.; threo-D-isoleucine was from California Biochemical Corporation, Los Angeles, California, and erythro-D-isoleucine from Koch-Light, Colnbrook, Bucks., England. Isoleucines of suitable purity were not readily obtainable. One sample of erythro-D-isoleucine, labelled 'chromatographically pure', contained at least four components. The isomers used were examined for optical purity by polarimetry, and for freedom from their epimers by ion-exchange chromatography (Spackman, Stein \& Moore, 1958). None was completely pure by either criterion; a sample was accepted for use when it contained no amino acid other than isoleucine, when its specific rotation was such as to exclude the presence of more than $3 \%(\mathrm{w} / \mathrm{w})$ of an isomer of opposite configuration at the $\alpha$-carbon atom, and when no more than $3 \%(w / v)$ of its epimer (threo- in erythro-isoleucines, and vice versa) was detected. These limits represented the best quality obtainable for amino acids from commercial sources. The difficulties of obtaining steroeochemically pure isoleucines were discussed by Greenstein \& Winitz (1961).

Analytical methods. Except where otherwise stated, details of or references to the analytical methods used in this work were those given by Bishop et al. (1965). Cyclodepsipeptides were determined by the method there described, except that pure diethyl ether was used. Primary amino acids were determined by ion-exchange chromatography, kindly done by $\mathrm{Mr} \mathrm{M}$. Hatton by the use of an EEL Automatic Analyser (Evans Electroselenium Ltd., Halstead, Essex). Quantitative paper chromatography of $\mathrm{N}$-methyl-leucine and other amino acids was done as described for isoleucine by Riches, Rothwell \& Russell (1966), with pure amino acids as standards. The sensitivity was lower for $N$-methyl-leucine (see Russell, $1960 \mathrm{~b}$ ), but satisfactory results were obtained in the range $0 \cdot 05-0 \cdot 2 \mu$ mole.

Hydrolysis of cyclodepsipeptides was done in concentrated hydrochloric acid + glacial acetic acid $\left(1+1\right.$, by vol.) at $110^{\circ}$ for $48 \mathrm{hr}$, and the excess of acid was removed in vacuo over potassium hydroxide. The molecular weight of sporidesmolide I is 638; to provide solutions of suitable concentration the hydrolysates were reconstituted to contain the products from $6 \cdot 38 \mu \mathrm{g}$./ml. for paper chromatography of amino acids or $638 \mu \mathrm{g} . / \mathrm{ml}$. for ion-exchange chromatography. Alternatively, the dried residues were extracted with ether for investigation of the hydroxy acids by paper chromatography as previously described (Russell, 1962).

Partial hydrazinolysis of cyclodepsipeptides was done, and the products were examined by thin-layer chromatography, as described elsewhere (Russell, 1965 $a, b$ ).

Infrared spectra were measured for paraffin mulls with an Infracord 137 spectrophotometer (Perkin-Elmer, Norwalk, Connecticut, U.S.A.), using cyclodepsipeptide samples dried in vacuo at $110^{\circ}$. 


\section{RESULTS}

Pithomyces chartarum grew well on the standardized potato + carrot medium, or on the defined medium + yeast extract. Isoleucine isomers did not greatly affect growth. The 'natural' isomer, erythro-L-isoleucine, was slightly but consistently stimulatory, whereas the other, 'unnatural', isomers had a slight inhibitory effect (Table 1).

The effects of the various isomers on the number of spores produced in culture were similar to their effects upon growth. Thus, while the 'unnatural' isomers were without effect on sporulation, the presence of erythro-L-isoleucine in the medium resulted in a twofold increase in spore number (Table 2).

Table 1. Effects of stereoisomeric isoleucines* on growth in Pithomyces chartarum grown in surface culture for 14 days at $25^{\circ}$ on potato + carrot extract

\begin{tabular}{|c|c|c|c|c|c|c|c|}
\hline \multirow{3}{*}{ Isomer } & \multicolumn{6}{|c|}{ Expt. no. } & \multirow{3}{*}{$\begin{array}{c}\text { Mean } \\
(\text { control }=100)\end{array}$} \\
\hline & $\mathrm{F} \dagger$ & $54 \ddagger$ & 59 & 76 & 77 & 90 & \\
\hline & \multicolumn{6}{|c|}{ Dry wt. (mg./ml.) } & \\
\hline Control & $4 \cdot 8$ & $3 \cdot 95$ & $2 \cdot 7$ & 6.5 & $6 \cdot 5$ & $6 \cdot 4$ & 100 \\
\hline Erythro-L & $5 \cdot 2$ & $5 \cdot 4$ & $3 \cdot 7$ & - & - & $7 \cdot 6$ & 125 \\
\hline Erythro-D & - & - & - & $5 \cdot 7$ & $5 \cdot 6$ & $5 \cdot 1$ & 85 \\
\hline Threo-L & $4 \cdot 0$ & $5 \cdot 2$ & $2 \cdot 1$ & 一 & - & $5 \cdot 1$ & 93 \\
\hline Threo-D & $3 \cdot 4$ & $3 \cdot \overline{1}$ & $3 \cdot 2$ & 一 & - & $7 \cdot 4$ & 95 \\
\hline
\end{tabular}

Table 2. Effects of stereoisomeric isoleucines* on sporulation in Pithomyces chartarum in surface culture for 14 days at $25^{\circ}$ on potato + carrot extract

\begin{tabular}{|c|c|c|c|c|c|c|c|}
\hline \multirow[b]{3}{*}{ Isomer } & \multicolumn{6}{|c|}{ Expt. no. } & \multirow[b]{3}{*}{$\begin{array}{c}\text { Mean (con- } \\
\text { trol }=10 \text { ) }\end{array}$} \\
\hline & $\mathrm{F} \dagger$ & $54 \sharp$ & 59 & 76 & 90 & $90 \mathrm{e}$ & \\
\hline & \multicolumn{6}{|c|}{ Spores $\left(\times 10^{-5} / \mathrm{ml}.\right)$} & \\
\hline Control & 40 & 19 & 7 & 44 & 26 & 26 & 100 \\
\hline Erythro-L & 44 & 25 & 26 & 一 & 47 & 58 & 205 \\
\hline Erythro-D & - & - & 一 & 49 & 24 & - & 102 \\
\hline Threo-L & 29 & 23 & 10 & - & 25 & - & 108 \\
\hline Threo-D & 26 & 13 & 7 & - & 36 & - & 93 \\
\hline & $/ \mathrm{m}$ & das & vth. & ines & $m+$ & xtract & \\
\hline
\end{tabular}

In estimating sporidesmolide yields, the ether used to purify the crude sporidesmolide residues was not previously saturated with the normal sporidesmolide mixture. The same modification was used, but by an oversight was not mentioned, by Bertaud et al. (1963). The object was to avoid contamination of those sporidesmolides being isolated with sporidesmolides produced under normal conditions, since these two were expected, and indeed were found, to differ in composition. Differences in composition might produce differences in ether-solubility. The method therefore did not necessarily 
give the true yields of sporidesmolides, but it did allow a comparison of the present results with those of Bertaud et al. (1963).

When sporidesmolide yields were determined in this way, all four stereoisomeric isoleucines were inhibitory. The inhibition produced by the D-isomers was only slight, whereas the L-isomers had a marked effect. However, because of the solubility considerations mentioned above, it could not be assumed that the yields found were true yields. That they were, in fact, too low, was demonstrated by thin-layer chromatography of the ether extracts (Bishop et al. 1965) which showed the presence therein of sporidesmolides. These extracts were therefore evaporated to dryness and the residues extracted with $n$-hexane. Thin-layer chromatography of the hexane extracts showed that sporidesmolides were absent. The hexane-insoluble residues were dissolved in chloroform and purified by percolation through charcoal and aluminum oxide (Bishop et al. 1965), and from this treatment further amounts of sporidesmolides were isolated. Absence of other types of compound was verified by thin-layer chromatography. In Table 3 all the individual yields listed are uncorrected for ether-solubility. The mean yields, expressed as percentage of the control values, are given both uncorrected and corrected. The effect of the correction is small except for threo-L-isoleucine; sporidesmolides produced on media containing this isomer were more soluble in ether than in the other cases. The corrected yields also demonstrated that both D-isomers were very weakly, if at all, inhibitory to sporidesmolide biosynthesis.

Table 3. Effects of stereoisomeric isoleucines* on sporidesmolide yield in Pithomyces chartarum

\begin{tabular}{|c|c|c|c|c|c|c|c|}
\hline \multirow[b]{2}{*}{ Isomer } & \multicolumn{5}{|c|}{ Sporidesmolides (mg. $/ 1.) \dagger$ in expt. no. } & \multicolumn{2}{|c|}{$\begin{array}{c}\text { Mean } \\
\text { (control }=100)\end{array}$} \\
\hline & $F$ & 59 & 76 & 77 & 90 & Uncorr. $\dagger$ & Corr. \\
\hline Control & 109 & 54 & 140 & 145 & 279 & 100 & 100 \\
\hline Erythro-L & 54 & 32 & - & - & 179 & 58 & 68 \\
\hline Erythro-D & - & - & 153 & 135 & 163 & 87 & 92 \\
\hline Threo-L & 22 & 3 & - & - & 75 & 18 & 29 \\
\hline Threo-D & 83 & 52 & - & - & 215 & 83 & 96 \\
\hline
\end{tabular}

* $5 \mathrm{mg} . / \mathrm{ml}$. $\dagger$ Results uncorrected for solubility in ether. $\ddagger$ Results corrected for solubility in ether.

Comparison of ether-soluble and ether-insoluble sporidesmolides by thin-layer chromatography revealed no difference in the number or relative intensities of the spots. It seems that the solubilities of individual sporidesmolides are not manifested independently, but depend on the composition of the mixture.

It has been established that in Pithomyces chartarum most if not all of the sporidesmolide mixture present occurs on the spores, and when spores are not formed, sporidesmolides cannot be detected in cultures (Bertaud et al. 1963). Inhibition of sporidesmolide biosynthesis by isoleucines might therefore occur by depression of sporulation, or by decrease in the amount synthesized per spore. The yields were therefore recalculated as mg. sporidesmolides $/ 10^{8}$ spores. The amount of sporidesmolides per spore in control cultures was very variable. Addition of either D-isomer did not inhibit sporidesmolide biosynthesis to a significant extent in terms either of total synthesis or of amount synthesized per spore. The depression produced by the 
L-isomers resulted largely from a decrease in the amount synthesized per spore, and not from inhibition of sporulation (Table 4).

When the solubility correction was applied, erythro-L-isoleucine was found to inhibit sporidesmolide synthesis per spore more than threo-L-isoleucine.

The amounts of isoleucine remaining in the media at harvest were determined by paper chromatography. Erythro-L-isoleucine, a protein constituent presumably abundant in natural substrates, was completely utilized. It is doubtful whether any

Table 4. Effects of stereoisomeric isoleucines* on sporidesmolides/spore in Pithomyces chartarum

\begin{tabular}{|c|c|c|c|c|c|c|c|}
\hline \multirow[b]{2}{*}{ Isomer } & \multicolumn{5}{|c|}{ Sporidesmolides/spore $\dagger$ in expt. no. } & \multicolumn{2}{|c|}{ Mean $\ddagger$} \\
\hline & $\mathrm{F}$ & 59 & 76 & 90 & $90 \mathrm{e}$ & Uncorr. & Corr. \\
\hline $\begin{array}{l}\text { Control } \\
\text { (mg. } / 10^{8} \text { spores) }\end{array}$ & $\begin{array}{l}100 \\
(2 \cdot 7)\end{array}$ & $\begin{array}{l}100 \\
(7 \cdot 7)\end{array}$ & $\begin{array}{l}100 \\
(3 \cdot 2)\end{array}$ & $\begin{array}{c}100 \\
(10 \cdot 7)\end{array}$ & $\begin{array}{c}100 \\
(10 \cdot 7)\end{array}$ & 100 & 100 \\
\hline Erythro-L & 45 & 16 & - & 35 & 30 & 33 & 39 \\
\hline Erythro-D & - & - & 98 & 63 & - & 81 & 85 \\
\hline Threo-L & 27 & 4 & - & 28 & - & 30 & 49 \\
\hline Threo-D & 117 & 96 & - & 55 & - & 89 & 102 \\
\hline
\end{tabular}

* $5 \mathrm{mg} . / \mathrm{ml}$. † Expressed as a percentage of the value for control cultures. $\ddagger$ Results given uncorrected, and corrected, for solubility in ether.

Table 5. Utilization of stereoisomeric isoleucines* by Pithomyces chartarum growing for 2 weeks at $25^{\circ}$ on potato + carrot extract

\begin{tabular}{lccc} 
Isomer added & $\begin{array}{c}\text { Amount } \\
\text { utilized }\end{array}$ & \multicolumn{2}{c}{$\begin{array}{c}\text { Composition of residual } \\
\text { amino acid (\%) }\end{array}$} \\
\cline { 2 - 3 } Erythro-L & $(\%)$ & Erythro & Threo \\
Erythro-D & 100 & - & - \\
Threo-L & 29 & 79 & 21 \\
Threo-D & 20 & 1 & 99 \\
& 82 & 6.5 & 93.5 \\
& * Initial concentration $5 \mathrm{mg} . / \mathrm{ml}$.
\end{tabular}

other isomer is normally encountered in more than traces by the organism. Threo-L isoleucine and erythro-D-isoleucine were poorly but threo-D-isoleucine was wel utilized (Table 5). A possible reason for this difference was revealed by analysis ot the composition of the residual isoleucines using ion-exchange chromatography. Som conversion of erythro-D-isoleucine to a threo-isomer had occurred. No instance o: inversion of an isoleucine isomer solely at the $\beta$-carbon atom is known; this changt then may be presumed to result from inversion at the $\alpha$-carbon atom, producin threo-L-isoleucine, an isomer which is also poorly utilized. If we postulate, to accoun for such an inversion, that Pithomyces chartarum produces an extracellular enzyms that racemizes D-amino acids then it is likely that threo-D-isoleucine in the mediun would be converted by such an enzyme to its well-utilized epimer, erythro-L-isoleucine and would thus appear to be well utilized. Some erythro-isoleucine was indeer present when cultures containing threo-D-isoleucine were harvested. Although thi amount was less than was present as impurity at the outset, erythro-L-isoleucine $\mathbf{i}$ 
well utilized and would not be expected to persist in the medium. The evidence is therefore in favour of its having been produced by epimerization of threo-D-isoleucine during the incubation period.

\section{Analysis of the effects of stereoisomeric isoleucines on sporidesmolide composition}

On unsupplemented growth media, Pithomyces chartarum produces a mixture of sporidesmolides. Mixtures were also formed on media containing amino acid supplements. No attempt was made to isolate the separate components, but physical and chemical methods were used to investigate the mixtures; the results gave evidence of their composition. Some notes on the methods used are given below; the results are presented in Table 6 .

Melting point. Each known sporidesmolide has a characteristic melting point, but measurement of this constant is of little value in characterizing mixtures, which tend to melt at intermediate temperatures. Sporidesmolide II has a very much lower m.p. than sporidesmolide I, and a binary mixture with m.p. close to that of sporidesmolide II should contain little sporidesmolide I.

Specific rotation. The specific rotations of the known sporidesmolides are too similar for this constant to be used in analysing mixtures of unknown composition. The measurements provided good evidence that the mixtures consisted largely of sporidesmolides since all, including sporidesmolides from control cultures, gave values for this constant of about $-200^{\circ}$ in chloroform solution.

Thin-layer chromatography. Sporidesmolides I and II were not resolved in the system used (Bishop et al. 1965) nor in any of several others tried. The method indicated the presence or absence of sporidesmolides III and IV, and permitted the degree of complexity of the mixtures to be assessed.

Infrared spectroscopy. All the known sporidesmolides have very similar infrared spectra. During this work it was found that each had a weak absorption maximum in the $11-12 \mu$ region. The precise wavelength of this absorption maximum was characteristic of the particular sporidesmolide. Measurement of the whole spectrum was used to confirm the preponderance in the mixtures of compounds with the general sporidesmolide structure. Measurement of the position of the absorption maxima at $11-12 \mu$ provided evidence as to the nature of the major components.

Amino acid analysis. Sporidesmolides I, II and III all differ in their amino composition (Table 6). Amino acid analysis of mixtures is therefore a guide to their composition. When these analyses were made by ion-exchange chromatography, significant quantities of erythro-isoleucine were detected. No sporidesmolide which contains this amino acid has yet been isolated. A threo-D-isoleucine residue is present in sporidesmolide II, and Shemyakin et al. (1963) reported that acid hydrolysis of sporidesmolide II was accompanied by slight epimerization of this amino acid. In the present work the ratio of erythro- to total isoleucine was different in hydrolysates of sporidesmolides from different treatments, although the hydrolysates were prepared at the same time and under identical conditions. Moreover, in one case $38 \%$ of the total isoleucine was erythro-isoleucine. It was concluded that the presence of erythroisoleucine in hydrolysates was not solely the result of epimerization during hydrolysis, but that contrary to a previous report based on paper chromatographic examination of hydrolysates (Russell, 1965c), erythro-isoleucine residues occurred in $P$. chartarum sporidesmolides. 


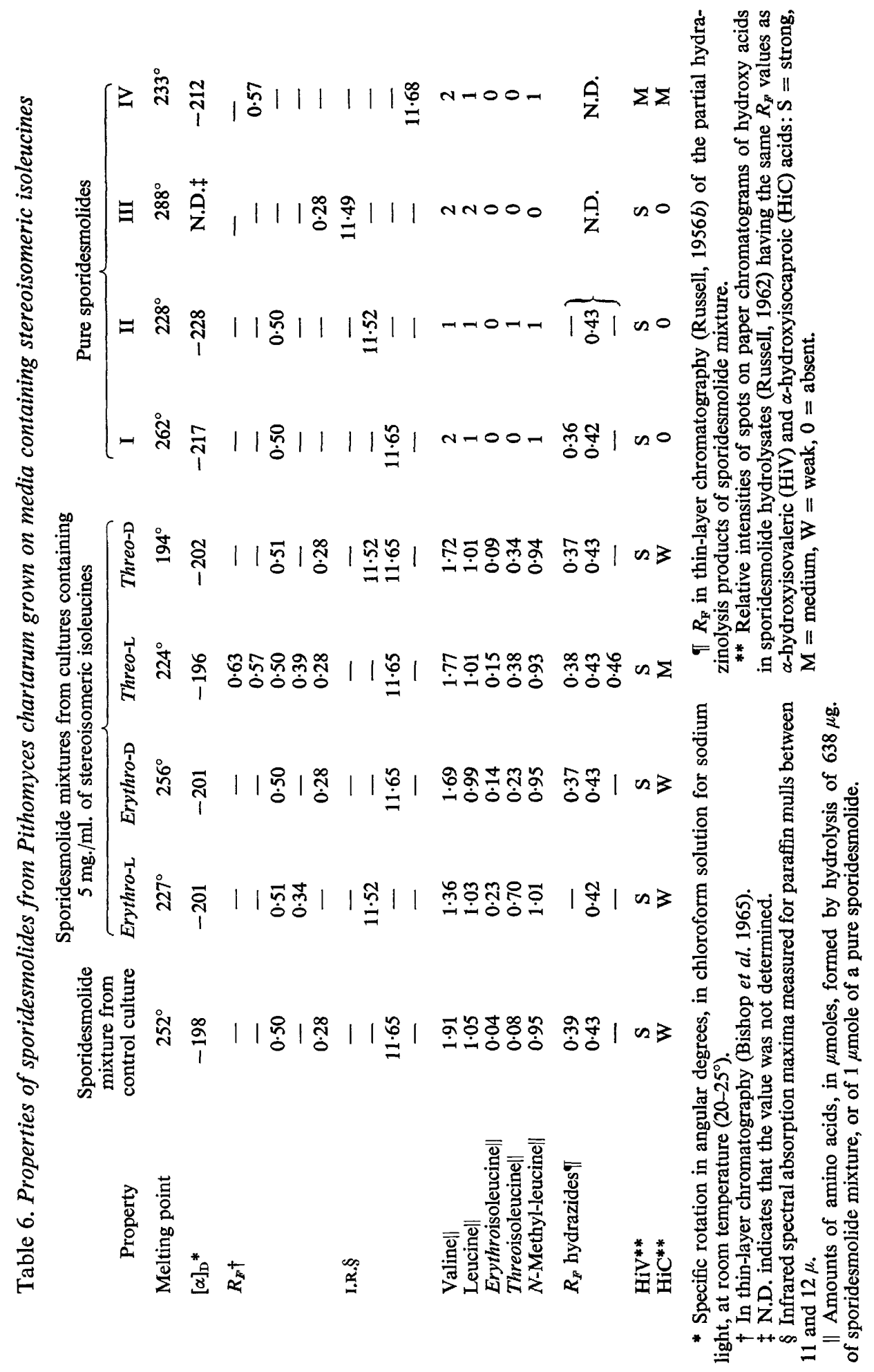


Hydroxy acid composition. Residues of $\alpha$-hydroxyisovaleric acid are present in sporidesmolides I, II and III. When hydrolysates of control sporidesmolides were examined by vapour-phase chromatography (kindly done by Dr D. G. Bishop; see Bishop \& Russell, 1967; the presence of $\alpha$-hydroxyisocaproic acid was detected. This acid, one residue of which is present in sporidesmolide IV from Pithomyces maydicus (Bishop \& Russell, 1967; Ovchinnikov et al. 1966) constituted up to $10 \%$ (w/v) of the total hydroxyacids of sporidesmolides from three different isolates of $P$. chartarum. Hydrolysates of the sporidesmolide samples were examined by paper chromatography, and the amounts of the two hydroxyacids were estimated visually. The method did not distinguish between $\alpha$-hydroxyisocaproic and the isomeric $\alpha$-hydroxy- $\beta$-methylvaleric acid.

Hydrazide analysis. Partial hydrazinolysis of sporidesmolide I gives the hydrazides of sporidesmolic acids A and B. These were resolved by thin-layer chromatography. The two hydrazides produced by partial hydrazinolysis of sporidesmolide II were not resolved, but gave a single spot with the $R_{F}$ value of sporidesmolic acid B hydrazide. This technique was therefore useful in demonstrating the absence of sporidesmolide I.

\section{Composition of sporidesmolides from individual treatments}

Control cultures. By physical criteria the sporidesmolides from control cultures were identical with those previously isolated from Pithomyces chartarum grown on unsupplemented media. The amino acid analysis was similar to that previously reported (Russell, Synge, Taylor \& White, 1962). The total isoleucine present in hydrolysates was $0.12 \mu$ moles $/ 638 \mu \mathrm{g}$., of which $33 \%$ was erythro-isoleucine.

Cultures containing erythro-L-isoleucine. The presence of erythro-L-isoleucine in the culture medium inhibited synthesis of sporidesmolides I and III. The infrared spectrum of the product isolated was identical with that of sporidesmolide II. A small amount of another compound was revealed by thin-layer chromatography, and hydrolysates contained more valine and less threo-isoleucine than would be released on hydrolysis of pure sporidesmolide II. The total isoleucine released was very much higher $(0.93 \mu$ moles $/ 638 \mu \mathrm{g}$., $25 \%$ erythro $)$ than in the controls. The evidence is consistent with the interpretation that Pithomyces chartarum, grown on medium containing erythro-L-isoleucine, produces mainly sporidesmolide II.

Cultures containing erythro-D-isoleucine. Sporidesmolides synthesized on medium containing this isomer were indistinguishable from those from control cultures except by amino acid analysis. Hydrolysates contained more isoleucine $(0.43 \mu \mathrm{moles} / 638 \mu \mathrm{g}$., $38 \%$ erythro) and less valine than control hydrolysates. The evidence is consistent with the interpretation that the organism produced the same sporidesmolides whether grown in the presence or in the absence of erythro-D-isoleucine, but that this amino acid altered slightly the proportions of the components in favour of those containing isoleucine, particularly one or both erythro-isomers.

Cultures containing threo-L-isoleucine. The presence of this isomer in the medium resulted in production of a complex mixture of sporidesmolides. This experiment demonstrated the value of thin-layer chromatography for investigating sporidesmolide mixtures. Five components were resolved by this means, whilst the infrared spectrum differed from that of the control sporidesmolides only in that the major peaks were broader. Sporidesmolides produced on this treatment liberated on hydrolysis con- 
siderable amounts of isoleucine $(0.53 \mu$ moles $/ 638 \mu \mathrm{g}$., $28 \%$ erythro $)$, and about twice as much leucic acid was present as in control hydrolysates. From the results of thin-layer chromatography, all four known sporidesmolides could have been present in the mixture in addition to two unknown ones.

Cultures containing threo-D-isoleucine. Thin-layer chromatograms of sporidesmolides from control and threo-D-isoleucine-enriched cultures were indistinguishable, and there was no difference in the m.p., in specific rotation or in the results of hydrazide or hydroxyacid analysis. However, the infrared spectrum of the product from treated cultures showed absorption maxima characteristic of both sporidesmolide $I$ and sporidesmolide II. The hydrolysate contained considerable amounts of isoleucine $(0.43 \mu \mathrm{mole} / 638 \mu \mathrm{g}$., $21 \%$ erythro $)$. I infer that threo-D-isoleucine did not affect the nature of sporidesmolides produced by Pithomyces chartarum, but that it altered their proportions in favour of those containing isoleucine, particularly of the threoconfiguration.

\section{DISCUSSION}

When a D-amino acid is utilized by a micro-organism at a slower rate than its L-isomer it may be difficult to decide whether the D-isomer is itself absorbed, or whether it is first inverted by an extracellular enzyme. The present experiments provide an instance of how such extracellular racemization may sometimes be simply detected. Both erythro-D- and threo-L-isoleucine are poorly absorbed and, since they are epimers, the extracellular conversion of one into the other is readily detected by chromatography. This and other epimeric pairs could be useful in detecting the secretion of extracellular amino acid-racemizing enzymes, and more generally in investigating biosynthetic mechanisms in which inversion occurs or is suspected.

In the present instance, the effects of D-isoleucines on sporidesmolide biosynthesis are largely explicable as due to their slow extracellular conversion to the corresponding L-epimers. This is consistent with the observation that ${ }^{14} \mathrm{C}$-D-valine was utilized by Pithomyces chartarum for sporidesmolide I biosynthesis with about one-third of the efficiency of the L-isomer, but that the distribution of radioactivity in sporidesmolide I was identical for both isomers (W. D. Bennett \& G. W. Butler, personal communication).

The whole of the sporidesmolide I molecule, except for the $N$-methyl group, may be derived from L-valine and L-leucine supplied in the medium (Butler et al. 1962). Examples of antagonism, both between valine and isoleucine, and between leucine and isoleucine, have been reported (Meister, 1965). Each of the four stereoisomeric isoleucines might act as antagonist towards formation of any or all of the six residues of sporidesmolide I. Even if the effects of the D-isomers are due largely to their L-epimers, the possibilities are still numerous. The complexity of the situation is reflected in the effects of threo-L-isoleucine, in the presence of which at least five different sporidesmolides, or closely related compounds, were synthesized. Not only are the identities of these compounds unknown; it is also uncertain whether formation of any or all of them was induced by the amino acid, or whether they are all present, some in trace amount, in the sporidesmolide mixture normally produced by Pithomyces chartarum. The presence of erythro-isoleucine and $\alpha$-hydroxyisocaproic acid in hydrolysates of control sporidesmolide mixtures suggests that the latter may be the case, but much more chemical work is necessary before the effects of threo-L-isoleucine can be interpreted. 
A simple explanation of the effects of erythro-L-isoleucine is possible if we assume that biosynthesis of sporidesmolides I and II takes place by the same pathway; this is reasonable because they are homologues. On this hypothesis, the biosynthetic system will accept either L-valine for synthesis of sporidesmolide I containing a D-valine residue, or erythro-L-isoleucine for sporidesmolide II containing a threo-D-isoleucine residue. This is consistent with the known ability of Pithomyces chartarum to incorporate erythro-L-isoleucine into sporidesmolide isoleucine residues (Butler et al. 1962) and with the ability of L-valine completely to inhibit biosynthesis of sporidesmolides containing such residues (unpublished observations). With erythro-L-isoleucine present in excess, sporidesmolide II would similarly be synthesized to the exclusion of sporidesmolide I. In the presence of threo-D-isoleucine, extracellular inversion would produce only a slow supply of the erythro-L-isomer, so that a mixture of both sporidesmolides would result. This explanation, although attractive because of its simplicity, may well be wrong. The Pithomyces chartarum isolate used is almost certainly a heterokaryon (Dingley, 1962) and the effects of erythro-L-isoleucine, as well as of other isomers, might result from selection during growth. For this reason, little further progress in the study of sporidesmolide biosynthesis is likely until a highsporing homokaryotic strain is obtained.

The author thanks Dr A. Taylor for a generous gift of sporidesmolide II, and is pleased to acknowledge the assistance of Mrs P. Riches, Miss V. Ward and Mr A. Rothwell.

\section{REFERENCES}

Bertaud, W. S., Morice, I. M., Russell, D. W. \& TAYlor, A. (1963). The spore surface in Pithomyces chartarum. J. gen. Microbiol. 32, 385.

Bertaud, W. S., Probine, M. C., Shannon, J. S. \& Taylor, A. (1965). Isolation of a new depsipeptide from Pithomyces chartarum. Tetrahedron 21, 677.

Bishop, E. \& Russell, D. W. (1964). Chemotaxonomic use of depsipeptides: Pithomyces maydicus and $P$. chartarum. Biochem. J. 92, 19P.

Bishop, E. \& Russell, D. W. (1967). Isolation and structure of Sporidesmolide IV, a cyclohexadepsipeptide from Pithomyces maydicus. J. chem. Soc. (c) p. 634.

Bishor, E., Griffiths, H., Russell, D. W., WARD, V. \& Gartside, R. N. (1965). Spore surface depsipeptides in some Pithomyces species. J. gen. Microbiol. 38, 289.

Butler, G. W., Russell, D. W. \& ClaRKE, R. T. J. (1962). Incorporation of ${ }^{14}$ C-labelled amino acids into sporidesmolide I by Pithomyces chartarum (Sporidesmium bakeri). Biochim. biophys. Acta 58, 507.

Dingley, J. M. (1962). Pithomyces chartarum, its occurrence, morphology, and taxonomy. N.Z. J. agric. Res. 5, 49.

Done, J., Mortimer, P. H., Taylor, A. \& Russell, D. W. (1961). The production of sporidesmin and sporidesmolides by Pithomyces chartarum. J. gen. Microbiol. 29, 207.

Ellis, M. B. (1960). Dematiaceous hyphomycetes. 1. Mycol. Pap. no. 76.

Greenstein, J. P. \& Winitz, M. (1961). Chemistry of the Amino Acids, Vol. 3, p. 2044. New York: John Wiley and Sons, Inc.

Meister, A. (1965). Biochemistry of the Amino Acids, Vol. 2, p. 231. New York: Academic Press Inc.

Ovchinnikov, Yu. A., Kiryushinin, A. A. \& Shemyakin, M. M. (1966). Total synthesis of sporidesmolides III and IV. Zh. obshch. Khim. 36, 620 (in Russian).

Riches, P., Rothwell, A. \& Russell, D. W. (1966). The spore surface depsipeptide of Pithomyces sacchari. J gen. Microbiol. 46, 77.

Ross, D. J. (1960). A study of the physiology of Pithomyces chartarum (Berk. and Curt.) M. B. Ellis. 2. Some factors affecting sporulation in culture. N.Z. J. Sci. 3, 441.

RusSell, D. W. (1960a). Sporidesmolide I, a metabolic product of Sporidesmium bakeri Syd. Biochim. biophys. Acta 45, 411.

RusSELl, D. W. $(1960 b)$. Ninhydrin as a reagent for $N$-methyl-amino acids. J. Chromatog. 4, 251. 
Russell, D. W. (1962). Depsipeptides of Pithomyces chartarum: The structure of sporidesmolide I. J. chem. Soc. p. 753.

RusSELL, D. W. (1965a). Angolide, a naturally-occurring cyclotetradepsipeptide with a twelvemembered ring. J. chem. Soc. p. 4664.

Russell, D. W. $(1965 b)$. Paper electrophoresis and some other techniques for separating acyl hydrazides. J. Chromatog. 19, 199.

RUSSELL, D. W. (1965c). Effects of isoleucine stereoisomers on yield and composition of Pithomyces chartarum depsipeptides. Abstr. 2nd Meeting Fed. Europ. Biochem. Soc. no. A 9.

Russell, D. W., Macdonald, C. G. \& Shannon, J.S. (1964). The structure of the cyclohexadepsipeptide, sporidesmolide. III. Tetrahedron Letters p. 2759.

Russell, D. W., Synge, R. L. M., TAylor, A. \& White, E. P. (1962). Similarity of depsipeptides from Pithomyces chartarum and from pasture samples from "Facial Eczema" areas. J. chem. Soc. p. 554.

Shemyakin, M. M., Ovchinnikov, Yu. A., Ivanov, V. T., Kiryushkin, A. A. \& Khalilulina, K. KH. (1965). The structure and total synthesis of sporidesmolides I and II. Zh. obshch. Khim. 35, 1399 (in Russian).

Spackman, D. H., Stein, W. H. \& Moore, S. (1958). Automatic recording apparatus for use in the chromatography of amino acids. Analyt. Chem. 30, 1190.

VickERY, H. B. (1963). Addendum to 'Definitive Rules for the Nomenclature of Natural Amino Acids and Related Substances'. J. org. Chem. 28, 291. 